\title{
djb präsent
}

9. Januar: Auftakt 2008, DAV, Berlin; Teilnahme: Jutta Wagner, Präsidentin. 11. Januar: Neujahrsempfang der IHK Berlin; Teilnahme: Prof. (Asoc.) Dr. jur. Jutta Glock, Vorsitzende LV Berlin (Vors. LV BE).

14. Januar: Die Charta der Rechte hilfeund pflegebedürftiger Menschen - Praxisbeispiele und erste Erfahrungen, Leitstelle Altenpflege am Deutschen Zentrum für Altersfragen und BMFSFJ, Berlin; Teilnahme: Dr. Trude-Lotte Steinberg-Krupp, Mitglied der Kommission Ältere Menschen (K ÄM).

16. Januar: Rechtspolitischer Neujahrsempfang, Bundesministerin Zypries, Berlin; Teilnahme: Jutta Wagner, Präsidentin.

16. Januar: AGG und die Auswirkungen in der Praxis, Juristische Gesellschaft zu Berlin; Teilnahme: Prof. (Asoc.) Dr. jur. Jutta Glock (Vors. LV BE) als Referentin.

18. Januar: Mitgliederversammlung UNIFEM, Bonn; Teilnahme: Sabine Overkämping, Mitglied der Kommission Öffentliches Recht, Europa- und Völkerrecht (K5), Vorsitzende der UG Europa (K5 UG Eur).

18.-19. Januar: Kinderschutz und Kindeswohl im Sorge- und Umgangsrecht Entwicklungen, Probleme, Lösungsansätze, Zentrale Informationsstelle der Autonomen Frauenhäuser (ZIF) u.a., Frankfurt/M.; Teilnahme: Dr. Angelika Nake, Vorsitzende der Kommission Zivil-, Familien- und Erbrecht, Recht anderer Lebensgemeinschaften (K2); als Referentinnen: Dagmar Freudenberg, Vorsitzende der Kommission Gewalt gegen Frauen und Kinder (Vors. KGew); Dr. Susanne Nothhafft und Sabine Heinke (KGew).

22. Januar: Reihe Rechtsanwältinnen, Gottfried Wilhelm Leibniz-Universität Hannover, Teilnahme: Jutta Wagner, Präsidentin, mit einem Vortrag.

22. Januar: Arbeit ohne Grenzen?, FES, Berlin; Teilnahme: Dr. Katja Rodi (Vorsitzende K5).

23. Januar: Berufsbild Anwältin: Erfahrungen erfolgreicher Juristinnen, djb und Prof. Dr. Susanne Baer, Juristische Fakultät der Humboldt-Universität zu Berlin; Teilnahme: Esther Hartwich, Junge Juristin LV Berlin; als Referentinnen: Prof. (Asoc.) Dr. jur. Jutta Glock (Vors. LV BE); Jutta von Falkenhausen, M.P.A.

24. Januar: Runder Tisch zum Thema Gesetzliche Implementierung psychosozialer Prozessbegleitung, BMJ, Berlin; Teilnahme: Dagmar Freudenberg (Vors. KGew).

24. Januar: Neujahrsempfang, Münchener Anwaltsverein; Teilnahme: Renate Maltry, Vizepräsidentin.

25. Januar: Ladies Lunch im Deutschen Bundestag, FDP; Teilnahme: Jutta Wagner, Präsidentin.

26. Januar: Neujahrsempfang, frauenbetriebe e.V., Oldenburg; Teilnahme: Birgit Kersten, RGB-Vorstand; Carolin Honisch, Junge Juristin LV Niedersachsen.

2. Februar: Menschenrechte gestalten? Das Beispiel des Alternativberichts zur UN-Frauenrechtskonvention (CEDAW), Berlin; Teilnahme: Prof. Dr. Susanne Baer, LL.M., Dr. Katja Rodi (Vors. K5) und Prof. Dr. Beate Rudolf (K5) als Veranstalterinnen und Referentinnen; Dr. Beate Schöpp-Schilling als Referentin. 11. Februar: FGG-Reformgesetz, Teil I: Allgemeines Verfahrensrecht, BTRechtsausschuss, Berlin; Teilnahme: Dr. Angelika Nake (Vors. K2) als Sachverständige.

11.-15. Februar: „Gar nicht pflegeleicht? Pflegenotstand und die Perspektiven der Absicherung von Altersrisiken, Gesellschaft für politische Bildung e.V., Würzburg; Teilnahme: Christiane Schreiber, Vorsitzende Kommission Ältere Menschen (K Ält).

13. Februar: FGG-Reformgesetz, Teil II: Familiengerichtliches Verfahren, BTRechtsausschuss, Berlin; Teilnahme: Susanne Nothhafft (K Gew) als Vertreterin für das Dt. Jugendinstitut.

13. Februar: Öffentliches Hearing zu Bachelor- und Master-Modellen für die Juristenausbildung, Stifterverband für die deutsche Wissenschaft, Generalsekretär,
Bonn; Teilnahme: Anna Eberhard und Barbara Dittmann, Vertreterinnen der Mitglieder in Ausbildung.

18. Februar: Abschiedsempfang für Uta Fölster, AG Berlin Mitte; Teilnahme: Jutta Wagner, Präsidentin.

18. Februar: Fachtagung „Reform des Vergaberechts“, Fraktion Bündnis 90/ Die Grünen im Deutschen Bundestag, Berlin; Teilnahme: Prof. Dr. Elke Gurlit als Referentin.

18. Februar: Öffentliche Anhörung „Frauen in der Wissenschaft und Gender in der Forschung “, BT-Ausschuss Bildung und Forschung, Berlin; Teilnahme: Prof. Dr. Susanne Baer als eingeladene Expertin; Dr. Katja Rodi (Vors. K5).

20. Februar: Empfang für die neu in Hamburg zugelassenen Rechtsanwältinnen und Rechtsanwälte, Rechtsanwaltskammer Hamburg; Teilnahme: Katharina Feddersen, LL.M. (Vorstand LV HH stellt den djb vor.

25. Februar: Anhörung zum Berliner Vergabegesetz, Ausschuss für Wirtschaft, Technologie und Frauen, Abgeordnetenhaus von Berlin, Berlin; Teilnahme: Prof. (Asoc.) Dr. jur. Jutta Glock (Vors. LV BE) (für die SPD-Fraktion im Berliner Abgeordnetenhaus).

29. Februar und 1. März: Die Frau ist frei geboren und bleibt dem Manne gleich“. Entwicklung der Gleichberechtigung im geteilten und geeinten Deutschland“, FES und Forum Justizgeschichte e.V., Berlin; Teilnahme: Prof. Dr. Sibylle Raasch, Vorsitzende der Kommission Arbeits-, Gleichstellungs- und Wirtschaftsrecht (K1), Abschlusspodium; u.a.

29. Februar-1. März: 3. Deutscher Erbrechtstag, AG Erbrecht des Deutschen Anwaltvereins, Berlin; Teilnahme: Jutta Wagner, Präsidentin.

3. März: Wie viel besser müssen Frauen sein?, Tagesspiegel und Initiative Neue Soziale Marktwirtschaft, Berlin; Teilnahme: Jutta Wagner, Präsidentin.

6. März: Amtseinführung der Präsidentin des Schleswig-Holsteinischen Oberlandesgerichts Uta Fölster, Schleswig; Teilnahme: Margret Diwell, Präsidentin des 
Verfassungsgerichts Berlin; Prof. (Asoc.) Dr. jur. Jutta Glock (Vors. LV BE).

6. März: Internationaler Frauentag, Landesvertretung Bremen beim Bund; Teilnahme: Jutta Wagner, Präsidentin.

6. März: Frauen überall hin! 1918-2008: 90 Jahre Frauenwahlrecht, Bündnis 90/ Die Grünen-Bundestagsfraktion, Berlin; Teilnahme: Jutta Wagner, Präsidentin. 7. März: Die Rolle der Frauen im Nationalsozialismus und in der neuen rechten Szene, FES - Forum Politik und Gesellschaft, Berlin; Teilnahme: Anke Gimbal, Geschäftsführerin.

7. März: Frauenfrühstück anlässlich des Internationalen Frauentages, Die Botschafter von Dänemark, Finnland, Island, Norwegen und Schweden, Berlin; Teilnahme: Jutta Wagner, Präsidentin.

7.-8. März: Flüchtlingsstatus und Bleiberecht in der deutschen Rechtspraxis. Wie gelungen ist die Umsetzung der Qualifikationsrichtlinie?, Ev. Akademie Loccum in Kooperation mit der Niedersächsischen Fachkonferenz für Flüchtlingsfragen, Loccum; Teilnahme: mehrere Mitglieder K 5.

10. März: Verleihung Anita Augspurg Preis der Landeshauptstadt München; Teilnahme: Renate Maltry, Vizepräsidentin.

10. März: „Frauen im Blick“ (es präsentieren sich die Frauenverbände Hamburgs, u.a. auch der LV HH des djb), Hamburg; Teilnahme: Monika Born, LV HH; Sylke Pukatzki, Delegierte für den Deutschen Frauenrat (Del DFR), als Mitorganisatorin für den Landesfrauenrat Hamburg.

13. März: Kinderrechte ins Grundgesetz - mehr Gerechtigkeit für Kinder, SPDBundestagsfraktion, Berlin; Teilnahme: Barbara Helfert.

17. März: Die Würde älterer Menschen schützen - Prävention von Gewalt gegen ältere Menschen und Vernachlässigung, Europäische Kommission, Brüssel; Teilnahme: Christiane Schreiber (Vors. K Ält) und Sabine Kräuter-Stockton, (K Gew) als eingeladene Expertinnen.

2.-3. April: Alter schafft Neues - Auf dem Weg zur Beteiligungsgesellschaft?, BMFSFJ, Berlin; Teilnahme: Christiane Schreiber (Vors. K Ält).
4. April: Auftaktveranstaltung zum 67. Deutschen Juristentag, Dt. Juristentag e.V. und Erfurter Juristische Gesellschaft, Erfurt; Teilnahme: Christina Lorenz, LV Thüringen.

4. April: Konferenz: Soziale Arbeit mit Opfern und Tätern sexueller Gewalt, FH Köln, Recht würde helfen, Köln; Teilnahme: Dagmar Freudenberg (Vors. KGew) als Referentin „Von der Konfrontation zur Kooperation“ Die Zusammenarbeit von Polizei, Justiz und Sozialer Arbeit im Opferschutz am Beispiel der (sozialpädagogischen) Prozessbegleitung.

8. April: Gesetz zur Modernisierung des Vergaberechts, Bundesministerium für Wirtschaft und Technologie, Berlin; Teilnahme: Prof. (Asoc.) Dr. jur. Jutta Glock (Vors. LV BE).

8. April: Preisverleihung Wettbewerb „Kulturelle Vielfalt in der Ausbildung“, Migrationsbeauftragte Prof. Dr. Maria Böhmer, Berlin; Teilnahme: Jutta Wagner, Präsidentin.

8. April: Besprechung Kinderförderungsgesetz - KiföG, BMFSFJ, Berlin; Teilnahme: Prof. Dr. Kirsten Scheiwe (K 4)

10. April: 14. Sozialrechtsforum, Forschungsstelle für Sozialrecht und Sozialpolitik an der Universität Hamburg; Teilnahme: Antje Reimers (K ÄM).

15. April: 35 Jahre Bayerischer Landsfrauenausschuss, München; Teilnahme: Renate Maltry, Vizepräsidentin.

16. April: „Pflegende und sorgende Frauen und Männer - Aspekte einer künftigen Pflege im Spannungsfeld von Privatheit und Professionalität“, Hessische Landeszentrale für politische Bildung, Frankfurt/M.; Teilnahme: Christiane Schreiber (Vors. K Ält).

18. April: 1. Sitzung der Expertengruppe zu Mediation in Zivil- und Handelssachen, BMJ, Berlin; Teilnahme: Natascha Wesel (LV BE).

23. April: 2. Dialog der 3-teiligen Dialogreihe zum Thema „Ein Jahr Elterngeld: mehr Geburten, mehr aktive Väter, mehr Jobchancen für Mütter ein familienpolitisches Wundermittel?!“, Zukunftsforum Familie e.V., Berlin; Teilnahme: Dr. Christine Fuchsloch (K 4) als Referentin.
23. April: 1. Berliner Kongress „Wertegesellschaft als ökonomischer Faktor“, Antidiskriminierungsstelle des Bundes, Berlin; Teilnahme: Jutta Wagner, Präsidentin; Prof. (Asoc.) Dr. jur. Jutta Glock (Vors. LV BE).

29. April: Konsultation der PatientInnenverfügung, DFR, Berlin; Teilnahme: Sylke Pukatzki (Del DFR).

1. Maì: „Anwältinnen im Management - Gender Mainstreaming in Unternehmen, Anwaltskanzleien und Anwaltsorganisationen“, DAV ARGE Anwältinnen, Berlin; Teilnahme: Jutta Wagner, Präsidentin; Jutta von Falkenhausen als Referentin.

1.-3. Mai: 59. Deutscher Anwaltstag, DAV, Berlin; Teilnahme: Jutta Wagner, Präsidentin, als Ehrengast; Renate Maltry, Vizepräsidentin; Dr. Angelika Nake (Vors. K2).

5. Mai: Eröffnung des gemeinsamen Aus- und Fortbildungszentrums der Landesverwaltung Brandenburg in Königs Wusterhausen, Land Brandenburg; Teilnahme: Ramona Pisal, Vizepräsidentin.

5. Mai: European Violence Prevention in Health Network, 2nd Workshop on health responses to interpersonal violence - network and clinical solutions, education, surveillance, Hessisches Sozialministerium, Wiesbaden; Teilnahme: Nancy Gage-Lindner (K Gew) als Einladende und Referentin.

7. Mai: Anhörung zum Bündnis 90/Die Grünen-Entwurf „Mehr Frauen in die Aufsichtsräte“, BT Rechtsausschuss, Berlin; Teilnahme: Prof. (Asoc.) Dr. jur. Jutta Glock (Vors. LV BE) für Bündnis 90/Die Grünen.

7. Mai: „100 Jahre Frauen in der Politik“ - Vorträge und Empfang, FDP im Deutschen Bundestag, Berlin; Teilnahme: Jutta Wagner, Präsidentin; Dr. Kerstin Niethammer-Jürgens (K2).

8. Mai: Der Vertrag von Lissabon - Eine stärkere Grundlage für Europa?, ERA, Berlin; Teilnahme: Sabine Overkämping (K5, UG Eur). 